\title{
Comparison of canal transportation and centering ability of three different endodontic rotary systems
}

\author{
(an invitro study)
}

\begin{abstract}
Shakhawan Kadir Kadir ${ }^{(1)}$
Background and objectives: one of the most important steps for root canal treatment is biomechanical preparation, which is the key stage of endodontic treatment with a predictive success factor if performed properly to prevent canal transportation. The aim of the present study is to compare the canal transportation and centering ability of Rotary ProTaper next, two Shape and Pro Taper gold systems in simulated root canals to find better instrumentation technique for maintaining root canal geometry.

Methods: Thirty simulated curved canals of $35^{\circ}$ curvature manufactured in clear resin blocks were randomly assigned to 3 groups $(n=10)$ according to the system used for canal instrumentation: ProTaper next, two Shape and Pro Taper gold systems groups, Centering ability was evaluated; Pre-and postoperative images of the canals were taken at a standardized position by a digital camera with a resolution of 25 mega pixel. An assessment of the shape of the canal was determined using Microsoft office Visio program version 11.

Results: The result showed that Protaper Gold produce less transportation and remained better center in the canal than ProTaper Next and 2 shape. The difference in transportation between the ProTaper Gold and ProTaper Next systems was not statistically significant differences $(P>0.05)$ while between ProTaper Gold and 2 shape and between ProTaper Next and 2 shape were significant differences $(P<0.05)$.

Conclusion: Protaper Gold produce less transportation of the canal than ProTaper Next and 2 shape.
\end{abstract}

Keywords: Protaper Gold, ProTaper Next , 2 shape ,centering ability, Canal transportation.

\footnotetext{
${ }^{(1)}$ Department of Conservative Dentistry, College of Dentistry, Hawler Medical University, Erbil, Iraq. Correspondent name: -Shakhawan kadir Email:shakhawankadir@yahoo.com
}

\section{Introduction}

The root canal treatment success depends on proper cleaning, widening, and shaping of mechanical instrumentation includes both enlargement and shaping ${ }^{1}$, creating space for the delivery of irrigation and antibacterial medicaments in eradicating bacteria and eliminating bacterial by-products. Thus creating proper space for three-dimensional obturation. . ${ }^{2}$

In the 1980s, Nickel-titanium (NiTi) rotary files were developed and have been associated with shorter instrumentation time and efficient cutting when compared with hand Instruments. ${ }^{3}$

Nickel-titanium (NiTi) instruments have been reported to have a decreased tendency for canal transportation and better centering ability than stainless steel due to their greater elasticity. ${ }^{4}$ Manufacturers strives to improve NiTi instruments by changing their design and enhancing the structural alloy in an attempt to improve their mechanical performance.

The M-wire heat-treated alloy designed with rectangular cross-section design for greater strength. The inventive design's axis of rotation differs from the center of mass. As a result, only two points of the rectangular cross-section touch the canal wall at a time and this rotary 
file is manufactured by ( Dentsply Sirona, Ballaigues, Switzerland.)This file called ProTaper Next (PTN) that gives the file a snake-like swaggering movement as it advances into the root canal.The PTN system is consist of $\mathrm{X} 1(17 / .04), \mathrm{X} 2(25 / .06), \mathrm{X} 3$ $(30 / .07), \mathrm{X} 4(40 / .06)$, and $\mathrm{X} 5(50 / .06)$ files. $^{2}$

2Shape (TS; MicroMega, Besancon, France) is made of T-wire heat-treated alloy with an asymmetric triangular cross-section. The 2S system is composed of TS1 (25/.04), TS2 (25/.06), F35 (36/.06), and F40 (40/.04) files. ${ }^{6}$

ProTaper Gold (Dentsply Tulsa Dental, Tulsa, OK, USA) has been introduced. According to the manufacturer, ProTaper Gold instruments have the same geometry as that of ProTaper Universal (Dentsply Maillefer, Ballaigues, Switzerland) but offer increased flexibility. The manufacturer claims that the ProTaper Gold instruments have resistance to cyclic fatigue and maintain canal centering, especially when preparing curved canals. ${ }^{7}$

For assessment of centering ability and canal transportation a variety of strategies are accustomed to comparing pre- and postinstrumentation canal shape. The aim of the present study is to evaluate the centering ability of PTN, 2 Shape and Protaper Gold rotary files and canal transportation in terms of maintaining the original root canal configuration in a simulated tooth with curvature.

\section{Material and method}

Description of the Resin Block. Standardized thirty endodontic-training blocks

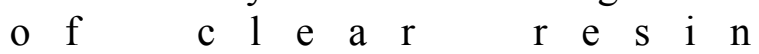
(Dentsply,Maillefer,Switzerland) were used, the block dimensions were $3 \times 1 \times 1 \mathrm{~cm}$, in the center of the block a simulated canal was manufactured so that it was parallel to one side of the block, and the canal communicates with external surface at the top \& bottom of the block. The total length of the canal was $18 \mathrm{~mm}$, the curvature was about $3 \mathrm{~mm}$ from the simulated apex, with a curvature of $35^{\circ}$ and a radius of $4 \mathrm{~mm}$, its size was equal to no.15.02 ISO K-files. ${ }^{8}$

Pre-operative preparation. The patency of each canal was checked using hand stainless steel K-file, .02, size \#15 and it introduced into the canal of each sample till it was just visible and felt tight at the apical foramen. The length of each canal was confirmed to be $18 \mathrm{~mm}$ by measuring the length of no. 15 stainless steel $\mathrm{k}$-file that was pre-curved to approximately $30^{\circ}$ to match the pre-operative canal shape using operator's finger. ${ }^{9}$

The resin blocks were divided randomly into 3 groups each had 10 blocks. Then using a digital camera(canon D5300 macro sigma $105 \mathrm{~mm}$ ) at a distance of $5.5 \mathrm{~cm}$, a pre -operative image with a resolution of 25 mega pixel was taken, for one canal that was filled, using a disposable syringe with 22 gauge needle, with red ink to enhance the image outline of the simulated canal, the image was taken and transferred to the computer later. Then the canal was irrigated with tap water using a disposable syringe with 22 gauge needle to remove the ink to prevent its dryness and blocking the canal. Then with the aid of Microsoft office Visio program version 11 , the pre-operative image of the resin block that was used in this study and was stored in the computer. ${ }^{(10)}$ The resin block was ready at that time to allow the instrumentation to start.

Group 1: Samples instrumented by Rotary ProTaper Next System. The files were used in an electric motor operated at $300 \mathrm{rpm}$ and at $2.5 \mathrm{~N}-\mathrm{cm}$ torques (X-Smart, Dentsply Tulsa Dental). The X1 file (17/.04, Dentsply Tulsa Dental) using a light coating Glyde File Prep as a lubricant was used with a brushing outstroke motion until resistance was felt in the canal. The file was then with- drawn, cleaned, and inspected for any defect before being reused. These procedures were repeated until the X1 file reached the WL. The same procedures were performed with the $\mathrm{X} 2$ file (25/.06, Dentsply Tulsa Dental). To standardize the irrigation protocol, Irrigation with $1 \mathrm{ml}$ normal saline was used after each instrument and $1 \mathrm{ml}$ after completing the 
instrumentation with disposable syringe and an open-end 22 gauge needle. After each instrument the needle was inserted until it reached the predetermined working length and retracted $2 \mathrm{~mm}$ before irrigation was applied. During instrumentation, the files were removed frequently from the canal and resin chips adhered to them was removed with a cotton roll dampened with tap water. After completion of canal preparation, the canal was filled with black ink using disposable syringe with 22 gauge needle and a post-operative image was captured and stored in the computer using the same steps of pre-operative image. ${ }^{10}$

Group 2: Samples instrumented by ProTaper Gold rotary file system. The instrumentation was started with ProTaper gold rotary file system. The instruments were driven at $300 \mathrm{rpm}$ with $2 \mathrm{~N} . \mathrm{cm}$ of torque. The previous guidelines were followed; after positioning of rubber stopper for all files, the sequence for preparation according to manufactured instruction was, S1 file which was used $2 \mathrm{~mm}$ shorter than the working length in a brushing motion for coronal one third with using a light coating Glyde File Prep that was applied to the file as a lubricant. Then S2 file which was used $2 \mathrm{~mm}$ shorter than the working length in a brushing motion for coronal two thirds with using a light coating Glyde File Prep as a lubricant. Finally F2 file was used for full working length using a light coating Glyde File Prep as a lubricant. The file was withdrawn once working length was reached by observing the position of the rubber stop on the file. Irrigation with $1 \mathrm{ml}$ normal saline was used after each instrument and $1 \mathrm{ml}$ after completing the instrumentation with disposable syringe and an open-end 22 gauge needle. After each instrument the needle was inserted until it reached the predetermined working length and retracted $2 \mathrm{~mm}$ before irrigation was applied.. During instrumentation, the files were removed frequently from the canal and resin chips adhered to them was removed with a cotton roll dampened with tap water. After completion of canal preparation, the canal was filled with black ink using disposable syringe with 22 gauge needle and a postoperative image was captured and stored in the computer using the same steps of preoperative image. ${ }^{8}$

Group 3: Samples instrumented by Rotary 2 Shape System. The instrumentation was started with 2 shape rotary file system. The instruments were driven at $300 \mathrm{rpm}$ with $1.2 \mathrm{~N} . \mathrm{cm}$ of torque values. Firstly TS1 was used followed by TS2 until the file reached the working length using a light coating Glyde File Prep as a lubricant. To standardize the irrigation protocol, Irrigation with $1 \mathrm{ml}$ normal saline was used after each instrument and $1 \mathrm{ml}$ after completing the instrumentation with disposable syringe and an open-end 22 gauge needle. After each instrument the needle was inserted until it reached the predetermined working length and retracted $2 \mathrm{~mm}$ before irrigation was applied. During instrumentation, the files were removed frequently from the canal and resin chips adhered to them was removed with a cotton roll dampened with tap water. After completion of canal preparation, the canal was filled with black ink using disposable syringe with 22 gauge needle and a postoperative image was captured and stored in the computer using the same steps of preoperative image. ${ }^{(8)}$

Measurement of Canal Transportation and centering ability, shifting of the long axis of the filed canal from the center line of the pre-operative canal at the apical third of the simulated canal were measured using the below formula by superimposing the pre and postoperative image, thus when the result of the formula was equal to zero it indicates optimum centering ability, while when the result of the formula was not equal to zero, it indicates the presence of transportation if measurements were done at the apical third of the simulated canal, whereas it indicates the presence of zipping if measurements were done at the apical 
exit, Also if the result was negative it means that the transportation or zipping was toward the outer (convex) side of the canal, whereas if the result was positive, it means that the transportation or zipping was toward the inner (concave) side of the canal. Also within each group, the number of the specimens that showed deviation toward the inner concave or the outer convex side was counted by measuring the mean of the parameter $\beta$ (with sign weather positive or negative) at all indicated levels within each specimen in the group, if the result was negative, it indicate deviation of the specimen as a consequence to the outer (convex) side, while if the result was positive, it indicate deviation of the specimen toward the inner (concave) side.

Parameter $\beta=[(\mathrm{D} 1-\mathrm{D} 2) / \mathrm{D}] \times 100$

$\mathrm{D} 1$ (X2-X1) being the distance between the edge of the original (pre-operative) canal and the edge of the filed (post-operative) canal on the concave side, D2 (Y2-Y1), being the distance between the edge of the original (pre-operative) canal and the edge of the field (post-operative) canal on the convex side, and $\mathrm{D}(\mathrm{X} 2+\mathrm{Y} 2)$, being the width of the filed canal. A value thus obtained for the parameter $B$, expressed in percentage terms, can be negative or positive. ${ }^{8}$ Statistical analysis: The collected data were analyzed by using SPSS (Statistical Package for Social Service) by version 22. for windows (SPSS, Chicago, Illinois, USA) using Analysis of variance (ANOVA) test to determine if there is significant difference among the means of groups followed by post hoc test. Statistical significant level was set at $\mathrm{P} £ 0.05$.

\section{Results}

The descriptive statistics for the mean values $\&$ the standard deviations of the parameter $\beta$ for the three instruments used for preparation of the simulated root canal samples that suffered transportation had been shown in the (Table 1) which show that the 2 shape system had higher mean value than that of parameter $\beta$ than that the Protaper next and Protaper Gold system.

By using one way ANOVA test, there was significant difference among the groups (Table 2 ).

Table 1: Descriptive Statistics of parameter $\beta$ that represent canal transportation of the three system used in the study

\begin{tabular}{|c|c|c|c|c|c|}
\hline & N & Minimum & Maximum & Mean & Std. Deviation \\
\hline PTN & 10 & 20.457 & 30.876 & 24.27610 & 3.856618 \\
\hline PTG & 10 & 18.459 & 26.996 & 22.77970 & 3.104500 \\
\hline TwOShape & 10 & 25.781 & 29.965 & 27.69450 & 1.431865 \\
\hline
\end{tabular}

Table 2: one way ANOVA for difference of canal transportation between three instruments systems used in the study

\begin{tabular}{|c|c|c|c|c|c|}
\hline & Sum of Squares & df & Mean Square & F & Sig. \\
\hline Between Groups & 126.933 & 2 & 63.467 & 7.168 & .003 \\
\hline Within Groups & 239.055 & 27 & 8.854 & & \\
\hline Total & 365.988 & 29 & & & \\
\hline
\end{tabular}

From the (Table 3), post hoc LSD test for the difference between each pair of the three groups. There is no significant difference between group PTN, and group PTG, while the difference between group PTN and 2 shape, but there is a highly significant difference between PTG and 2 shape. The direction of transportation whether toward inner side or outer side of the canal for all the samples tested is presented in (Table 4). 
Table 3: LSD test for the difference of canal transportation between each pair of the three groups of instrument systems used for preparation of the simulated root canal samples

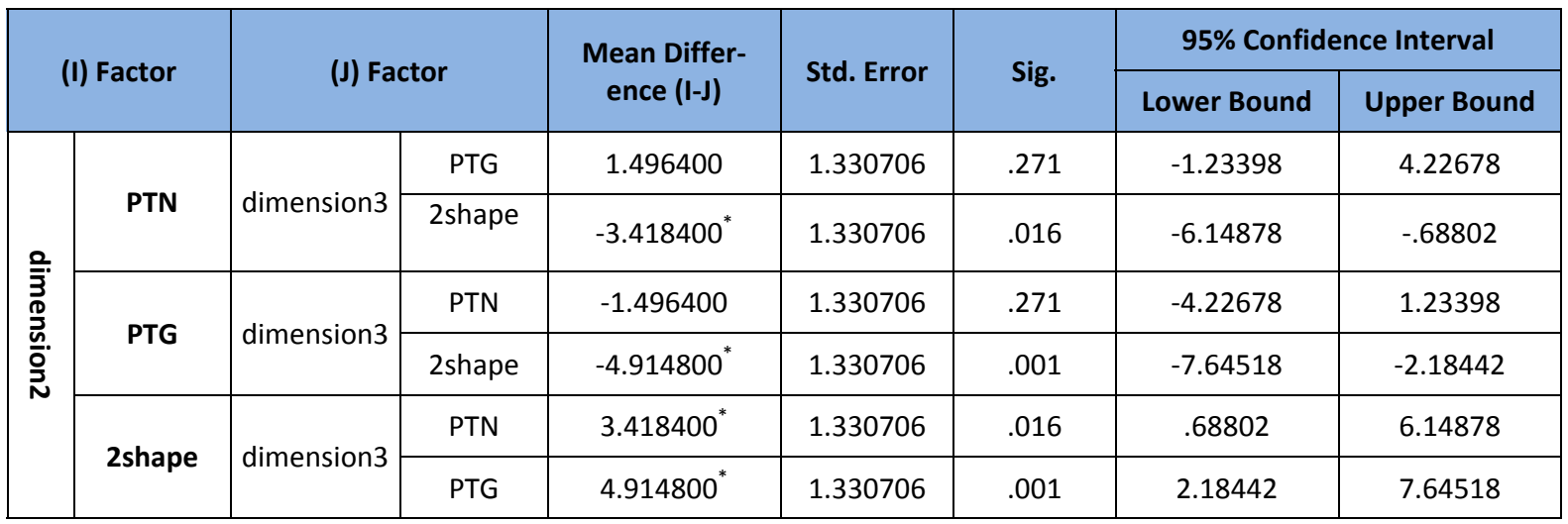

* The mean difference is significant at the 0.05 level.

Table 4: The direction of transportation whether toward inner side or outer side of the canal for three instrument systems used for preparation of the simulated root canal samples

\begin{tabular}{|c|c|c|}
\hline Instrument & No. of specimens deviated toward & No. of specimens deviated toward \\
\hline ProTaper next & 3 & 7 \\
\hline ProTaper Gold & 2 & 8 \\
\hline 2 Shape & 4 & 6 \\
\hline
\end{tabular}

\section{Discussion}

The simulated root canals which were used in this study allow a high degree of reproducibility and standardization for root canal diameter, length, curve angle, but do not reflect the clinical situation, additionally the difference in hardness between resin and dentin may affect the cutting behavior of instruments. The simulated canals have advantage over using radiographs in that it is straighter forward to compare pre and post preparation images. ${ }^{11,12}$

When a filing technique is applied to a curved root canal, severe transportation often occur, resulting in an hourglassshaped canal apically that may hamper complete obturation and could increase leakage. This condition may be exaggerated when the canal is very tiny. The convex (outer) wall of the apical portion of the curved root canal may be overinstrumented, removing valuable dentin. The concave (inner) wall may be untouched, and infection may remain and jeopardize apical healing, compromising the long-term success of treatment. ${ }^{13}$

The first aim of this study was to evaluate the differences in preparing curved simulated root canals using three different instruments: the classical ProTaper Gold technique using three files and the ProTaper Next and 2 shape technique using a two files. The classical ProTaper Gold technique uses variable taper shaping and finishing files to the working length of the preparation in an electric torque sensing motor at $300 \mathrm{rpm}$ in a continuously rotating motion. The design of the flutes together with a triangular cross section is claimed to remove dentin efficiently. The flexibility of Gold nickel titanium allows curved canals to be prepared with a reduced chance of procedural errors such as ledges or canal transportation. On the other hand it had a two-stage transformation behavior and high austenite finishing temperatures. 14, 15 While, the 2Shape (TS2; Micro-Mega, France) file is a new-generation file system produced with a proprietary heat treatment (T-Wire) that, according to the 
manufacturer, improves both the flexibility and cyclic fatigue resistance of the files. ${ }^{6}$ When comparing the three systems regarding the canal transportation, the result showed that Protaper Gold produce less transportation of the canal than ProTaper Next and 2 shape. The difference in transportation between the Gold and Next systems was not statistically significant while between Protaper Gold and 2 shape was significant and between Protaper next and 2 shape also significant, but all files have a tendency to straighten within the root canal regardless of the material used, Thus transportation results give an indication of how much material was removed with respect to the ideal preparation and indicate the direction of dentin removal. Transportation of the canal at this critical point can have two major drawbacks: first, the apical part of the canal on the inner side of the curvature may remain untouched and full of debris, and, second, it may lead to ledging or even a subsequent perforation. ${ }^{8}$

This result may be due to Early flaring of the coronal part of the root canal removes dentin constrictions, so that subsequent instruments do not bind short of the working length. ${ }^{16}$ ProTaper Gold rotary system has negative rake angles plus their greater cutting efficiency has been safely improved by balancing the pitch and helical angle in addition to a modified guiding instrument tip which in combination prevent the instruments from inadvertently screwing into the canal. ${ }^{17,18}$

Regarding pro taper next instruments are manufactured from M-Wire alloy, which has been proposed to improve file flexibility while retaining cutting efficiency. These files removed similar amounts of dentin because of the offset asymmetric design of this instrument which makes the files rotated in a unique asymmetric motion like a snake. ${ }^{19}$ Two Shape is a file system with variable cross section, presenting with 3 cutting edges. The 3 cutting edges guide the file down the canal, whereas the 2cutting-edge zone in the coronal portion offers optimal cutting. It has positive rake an- gle, variable, progressive pitch, and constant taper of $6 \%$, preparing the canal in shorter period of time. All these features in the file systems may contribute to production of more canal transportation. ${ }^{6}$

\section{Conclusion}

Within the limitation of this study canal preparation with Protaper Gold produce less transportation of the canal and better centering ability than ProTaper Next and 2 shape.

\section{Conflicts of interest}

The authors reported no conflict of interest.

\section{References}

1. Vallaeys K, Chevalier V, Arbab-Chirani R. Comparative analysis of canal transportation and centring ability of three $\mathrm{Ni}$-Ti rotary endodontic systems: Protaper ${ }^{\circledR}, \mathrm{MTwo}^{\circledR}$ and Revo-S ${ }^{\mathrm{TM}}$, assessed by micro-computed tomography. Odontology 2016; 104(1): 83-8.

2. Staffoli S, Özyürek T, Hadad A, Lvovsky A, Solomonov $M$, Azizi $H$, et al. Comparison of shaping ability of ProTaper Next and 2Shape nickeltitanium files in simulated severe curved canals. Giornale Italiano di Endodonzia. 2018 Nov;32 (2):52-6.

3. Mamede-Neto I, Borges AH, Guedes OA, de Oliveira D, Pedro FLM, Estrela C. Root Canal Transportation and Centering Ability of Nickel-Titanium Rotary Instruments in Mandibular Premolars Assessed Using Cone-Beam Computed Tomography. Open Dent J. 2017 Feb 14;11:71-8.

4. McSpadden JT. Rationales for rotary nickeltitanium instruments. Chattanooga, TN: NT Co; 1994.

5. Walton RE, Torabinejad M. Principles and practice of endodontics. Elsevier Health Sciences; 2014: 210.

6. MicroMega. The 2Shape brochure. Available at: http://www.denta.be/pdf/MicroMega-2shapeENG.pdf. 2017 (last accessed 10/2018).

7. Ruddle CJ, Machtou P, West JD. Endodontic canal preparation: New innovations in glide path management and shaping canals. Dent today. 2014;33:118-23.

8. Bakr DKh. Assessment of Novel Self-Adjusting File System (In Vitro Study) Ph.D. thesis Hawler Medical University/ College of dentistry. 2013

9. Ingle, J. and Bakland, L. (2002) Endodontics. 5th Edition, BC Decker, Hamilton. 
10. Calberson, F. L.; Deroose, C. A.; Hommez, G. M. \& De Moor, R. J. Shaping ability of ProTaper nickel -titanium files in simulated resin root canals. Int. Endod. J., 37(9):613-23, 2004.

11. Hülsmann M, Stryga F (1993) Comparison of root canal pre-paration using different automated devices and hand instrumentation. Journal of Endodontics 19, 141-5.

12. Javaheri HH1, Javaheri GHA comparison of three $\mathrm{Ni}$-Ti rotary instruments in apical transportation. J Endod. 2007 Mar;33(3):284-6.

13. Peters OA Current challenges and concepts in the preparation of root canal systems

J Endod. 2004 Aug;30(8):559-67

14. Hieawy A, Haapasalo $M$, Zhou $H$, et al. Phase transformation behavior and resistance to bending and cyclic fatigue of ProTaper Gold and ProTaper Universal instruments. J Endod. 2015;41:1134 -1138 .
15. Uygun AD, Kol E, Topcu MK, et al. Variations in cyclic fatigue resistance among ProTaper Gold, ProTaper Next and ProTaper Universal instruments at different levels. Int Endod J. 2016;49:494-499.

16. Pitt Ford ,T.R.,Rhodes,J.S.,Pitt Ford,H.E.,(2002) Endodontic problem solving in clinical practice. Martin DuntizLtd;1st edition.

17.Webber J and Machton P (2001) protaper: taking the curve with ease. www.Dentisply .co.uk/ articles.acessed on 12/5/2008.

18. Koch and Brave. Endodontic:Real word endo:design features of rotary files and how they affect clinical perform ance.www.oralhealthjournal.2002Feb;39-49

19.Elnaghy AM Cyclic fatigue resistance of ProTaper Next nickel-titanium rotary file Int Endod J. 2014 Nov;47(11):1034-9 